\title{
PARTICIPANTS AT THE ROUND TABLE MEETING
}

\section{CHAIRMAN}

\section{E.D. Bateman}

University of Cape Town, Cape Town, South Africa.

\section{PRESENTERS}

\section{P. Anderson}

University of Arkansas for Medical Sciences, Little Rock, AR, USA.

\section{R. Booker}

National Respiratory Training Centre, Warwick, UK.

K.R. Chapman

University Health Network, Toronto, ON, Canada.

\section{M.E. Hyland}

University of Plymouth, Plymouth, UK.

\section{S.P. Newman}

Scientific Consultant, Nottingham, UK.

\section{L.M. Osman}

Aberdeen Royal Infirmary, Aberdeen, UK.

C.S. Rand

Johns Hopkins School of Medicine, Baltimore, MD, USA.

\section{PARTICIPANTS}

P. Byron

Virginia Commonwealth University, Richmond, VA, USA.

R. Dahl

Åarhus University Hospital, Åarhus, Denmark.

\section{R. Hodder}

The Ottawa Hospital, Ottawa, ON, Canada.

J.C. Virchow

University Medical Clinic, Rostock, Germany.

\section{T.H. Voshaar}

Bethanien Hospital, Moers, Germany. 